\section{Advanced Liver Fibrosis Is Common in Patients With Type 2 Diabetes Followed in the Outpatient Setting: The Need for Systematic Screening}

Diabetes Care 2021;44:399-406 | https://doi.org/10.2337/dc20-1997

\section{OBJECTIVE}

Assess the prevalence of nonalcoholic fatty liver disease (NAFLD) and of liver fibrosis associated with nonalcoholic steatohepatitis in unselected patients with type 2 diabetes mellitus (T2DM).

\section{RESEARCH DESIGN AND METHODS}

A total of 561 patients with T2DM (age: $60 \pm 11$ years; BMI: $33.4 \pm 6.2 \mathrm{~kg} / \mathrm{m}^{2}$; and $\mathrm{HbA}_{1 \mathrm{c}}: 7.5 \pm 1.8 \%$ ) attending primary care or endocrinology outpatient clinics and unaware of having NAFLD were recruited. At the visit, volunteers were invited to be screened by elastography for steatosis and fibrosis by controlled attenuation parameter $(\geq 274 \mathrm{~dB} / \mathrm{m}$ ) and liver stiffness measurement (LSM; $\geq 7.0 \mathrm{kPa}$ ), respectively. Secondary causes of liver disease were ruled out. Diagnostic panels for prediction of advanced fibrosis, such as AST-to-platelet ratio index (APRI) and Fibrosis-4 (FIB-4) index, were also measured. A liver biopsy was performed if results were suggestive of fibrosis.

\section{RESULTS}

The prevalence of steatosis was $70 \%$ and of fibrosis $21 \%$ ( $L S M \geq 7.0 \mathrm{kPa}$ ). Moderate fibrosis (F2: LSM $\geq 8.2 \mathrm{kPa}$ ) was present in $6 \%$ and severe fibrosis or cirrhosis (F3-4: LSM $\geq 9.7 \mathrm{kPa}$ ) in $9 \%$, similar to that estimated by FIB-4 and APRI panels. Noninvasive testing was consistent with liver biopsy results. Elevated AST or ALT $\geq 40$ units/L was present in a minority of patients with steatosis ( $8 \%$ and $13 \%$, respectively) or with liver fibrosis ( $18 \%$ and $28 \%$, respectively). This suggests that AST/ALT alone are insufficient as initial screening. However, performance may be enhanced by imaging (e.g., transient elastography) and plasma diagnostic panels (e.g., FIB-4 and APRI).

\section{CONCLUSIONS}

Moderate-to-advanced fibrosis (F2 or higher), an established risk factor for cirrhosis and overall mortality, affects at least one out of six (15\%) patients with T2DM. These results support the American Diabetes Association guidelines to screen for clinically significant fibrosis in patients with T2DM with steatosis or elevated ALT.

Nonalcoholic fatty liver disease (NAFLD) is the most common chronic liver disease (1-3). The more severe form, known as nonalcoholic steatohepatitis (NASH), is characterized by predominantly lobular necroinflammation, with or without centrilobular fibrosis, which may progress to cirrhosis and even hepatocellular carcinoma
Romina Lomonaco, ${ }^{1}$

Eddison Godinez Leiva, ${ }^{1}$ Fernando Bril, ${ }^{1}$

Sulav Shrestha, ${ }^{1}$ Lydia Mansour, ${ }^{1}$

Jeff Budd, ${ }^{2}$ Jessica Portillo Romero, ${ }^{2}$

Siegfried Schmidt, ${ }^{3}$ Ku-Lang Chang, ${ }^{3}$

George Samraj, ${ }^{3}$ John Malaty, ${ }^{3}$

Katherine Huber, ${ }^{2}$ Pierre Bedossa, ${ }^{4}$

Srilaxmi Kalavalapalli, ${ }^{1}$ Jonathan Marte, ${ }^{1}$

Diana Barb, ${ }^{1}$ Danielle Poulton, ${ }^{1}$

Nada Fanous, ${ }^{1}$ and Kenneth Cusi, ${ }^{1,5}$

${ }^{1}$ Division of Endocrinology, Diabetes and Metabolism, University of Florida, Gainesville, FL

${ }^{2}$ Division of General Internal Medicine, University of Florida, Gainesville, FL

${ }^{3}$ Department of Family Medicine, University of Florida, Gainesville, FL

${ }^{4}$ Department of Pathology, Beaujon Hospital Paris Diderot University, Paris, France

${ }^{5}$ Division of Endocrinology, Diabetes and Metabolism, Malcom Randall VA Medical Center, Gainesville, $F L$

Corresponding author: Kenneth Cusi, kenneth .cusi@medicine.ufl.edu

Received 11 August 2020 and accepted 6 November 2020

This article contains supplementary material online at https://doi.org/10.2337/figshare.13230857.

This article is featured in a podcast available at https://www.diabetesjournals.org/content/diabetescore-update-podcasts.

(C) 2020 by the American Diabetes Association. Readers may use this article as long as the work is properly cited, the use is educational and not for profit, and the work is not altered. More information is available at https://www.diabetesjournals .org/content/license. 
$(4,5)$. In 2019, the American Diabetes Association (ADA) recommended that patients with type 2 diabetes mellitus (T2DM) with elevated plasma ALT levels or steatosis on liver ultrasound should be evaluated for NASH and liver fibrosis (6). The rationale for screening such patients is based on increasing evidence that NASH with advanced fibrosis is associated with liver-related and extrahepatic morbidity and mortality $(7,8)$. In addition, steatohepatitis is believed to be more progressive in patients with versus without $\operatorname{T2DM}(2,9,10)$. It has also been associated with an increased risk of cardiovascular disease, which is already increased in diabetes $(11,12)$. However, evidence to support such a recommendation in the U.S. is rather modest, as the true prevalence of NAFLD, and specifically of NASH with fibrosis, remains unclear. Establishing the magnitude of the epidemic would be the first step to develop a sound public health policy.

It is important to highlight that the focus of screening in NAFLD is to identify patients with liver fibrosis at a time in the natural history when intervention can potentially prevent the progression to decompensated cirrhosis. Several therapies today offer such benefit: weight loss by lifestyle intervention or bariatric surgery, agents such as vitamin E (in patients without diabetes), and pioglitazone or glucagon-like peptide 1 receptor agonists (GLP-1RAs) in patients either with or without diabetes (13-17). A liver biopsy remains as the gold standard to assess the severity of disease activity and stage of liver fibrosis $(5,6)$. However, it is not a practical first-line approach for routine screening, as it is associated with the risks of an invasive procedure and it is relatively costly and subject to interpretation challenges (e.g., variability of histological reading based on sample size, location of the liver biopsy, pathologist expertise, etc.) (18).

Given the limitations of a liver biopsy for case finding, screening for clinically significant fibrosis has been centered on using serum biomarkers or liver imaging. Several plasma biomarkers and diagnostic panels of liver fibrosis have been tested $(19,20)$. However, few studies have focused exclusively on patients with T2DM. Among them, a study from France by Jacqueminet et al. (21) and another in the U.S. (22) reported a rate of advanced fibrosis of $\sim 6 \%$ in patients with T2DM.
More recently, Repetto et al. (23) reported a slightly higher rate of advanced liver fibrosis (F3-4) in obese patients with T2DM. Overall, diagnostic panels are considered to have suboptimal sensitivity for identifying early stages of fibrosis (e.g., especially if plasma AST/ALT are not elevated) but have reasonable specificity to identify precirrhosis or cirrhosis (F3-4) $(4,5)$ and even predict liver-related events (24). They also do not appear to perform as well as in patients with diabetes presenting to primary care or endocrinology clinics (25). Among the imaging modalities, liver stiffness measurement (LSM) by vibration-controlled transient elastography (VCTE) (FibroScan; Echosens, Paris, France) has been the most widely used in gastroenterology and hepatology clinics. It uses a pulseecho ultrasound technique to quantify the speed of mechanically induced shear wave within liver tissue. LSM has been well validated to correlate with the severity of fibrosis $(4,5)$. Hepatic steatosis can also be quantified at the same time by measuring the ultrasonic attenuation of the echo wave, known as controlled attenuation parameter (CAP). While there are some reports from primary care settings on the prevalence of steatosis and fibrosis in Europe (26-29) and Asia (30-33), there is no such information from randomly screened patients with T2DM attending outpatient clinics in the U.S.

The aim of this study was to establish the prevalence of steatosis and moderateto-advanced fibrosis by transient elastography in unselected patients with T2DM attending a general internal medicine, family medicine, or endocrinology outpatient clinic. Participants as well as their clinicians were unaware of the possibility of having NAFLD. We felt that this information would address a knowledge gap and be critical to support (or not) the current ADA Standards of Care recommendations for the management of NAFLD in patients with T2DM (6).

\section{RESEARCH DESIGN AND METHODS}

\section{Participants}

A total of 561 participants were recruited from internal medicine, family medicine, and endocrinology clinics at the University of Florida (UF). Patients were recruited between September 2018 and July 2020. Patients included were adults between 21 and 79 years of age, legally competent to provide written informed consent, and had T2DM according to ADA guidelines (6).

Exclusion criteria were an established history of NAFLD, history of alcohol abuse ( $\geq 30 \mathrm{~g} /$ day for males and $\geq 20 \mathrm{~g} /$ day for females), type 1 diabetes, any liver disease other than NAFLD (i.e., hepatitis B or $C$, autoimmune hepatitis, hemochromatosis, drug-induced hepatitis, etc.), on medications that could induce hepatic steatosis (e.g., estrogen, amiodarone, methotrexate, raloxifene, glucocorticoids, etc.), being on pioglitazone or GLP-1RAs within 6 months prior to enrollment, pregnancy/lactation, presence of implanted electronic medical device (i.e., pacemaker, as VCTE cannot be performed), or participation in another clinical trial within the preceding 30 days. The study was approved by the UF Institutional Review Board.

\section{Study Design}

This was a cross-sectional study aimed at evaluating the prevalence of liver fibrosis (primary outcome) by LSM using VCTE and liver steatosis among adults without known NAFLD. At the time of the clinic visit, volunteers were invited by their clinician to be screened by transient elastography for steatosis and fibrosis by CAP ( $\geq 274 \mathrm{~dB} / \mathrm{m}$ ) and LSM ( $\geq 7.0 \mathrm{kPa})$, respectively. After the research team obtained informed consent, they underwent an in-depth medical history, screening for alcohol intake by an Alcohol Use Disorders Identification Test questionnaire (adopted by the World Health Organization) and secondary causes of liver disease by medical history, review of electronic medical records, physical examination, and laboratory testing. Diagnostic panels for the prediction of advanced fibrosis were also measured, such as the ASTto-platelet ratio index (APRI) and Fibrosis-4 (FIB-4) index. If the patient was not fasting for $\geq 3 \mathrm{~h}$, patients were enrolled but elastography was postponed to a second research visit. Blood work was also deferred to a second visit if not fasting for at least $10 \mathrm{~h}$. Participants were invited to have a liver biopsy if the noninvasive workup was suggestive of liver fibrosis.

\section{Study Measures \\ VCTE}

VCTE testing was performed in the clinic with a FibroScan equipped with both $M$ and XL probes (model 530 compact; Echosens). This was performed by experienced research-only operators trained and certified by the manufacturer. An automatic probe selection tool is embedded in the device 
software that recommends the appropriate probe for each patient according to the realtime assessment of the skin-to-liver capsule distance. They were placed in a supine position with the right arm fully abducted. Once the liver area was defined, measurements were performed by scanning the right liver lobe through an intercostal space. At least 10 measurements at the same spot were performed with one single probe on each participant. Simultaneous CAP and LSM were obtained. CAP measured liver ultrasonic attenuation at $3.5 \mathrm{MHz}$ on both $\mathrm{M}$ and $X L$ probes (signals being acquired by the FibroScan). The principle of CAP measurement has been described elsewhere (34). Results were computed only when the associated LSM was valid and using the same signals as the one used to measure liver stiffness. The performance characteristics of VCTE for the evaluation of NAFLD have been reported elsewhere (35). Measurement of LSM was considered reliable only if the interquartile range/median ratio was $<30 \%$ and success rate was $>60 \%$. Results of CAP and LSM were expressed in decibels per meter $(\mathrm{dB} / \mathrm{m})$ and kilopascals $(\mathrm{kPa})$, respectively.

Presence of liver fibrosis was defined as follows: mild (F1) if $L S M \geq 7.0-8.1 \mathrm{kPa}$, moderate fibrosis (F2) if $\geq 8.2-9.6 \mathrm{kPa}$, advanced fibrosis (F3) if $\geq 9.7-13.5 \mathrm{kPa}$, and cirrhosis (F4) if $\geq 13.6 \mathrm{kPa}$, according to a previous landmark study (36) and adopted in other epidemiological studies (27). Patients were considered to have steatosis (secondary outcome) based on FibroScan if CAP $\geq 274 \mathrm{~dB} / \mathrm{m}$ and divided into mild, moderate, and severe steatosis defined as CAP 274$289 \mathrm{~dB} / \mathrm{m}$ ( $\mathrm{S} 1$ = mild), 290-301 dB/m ( $\mathrm{S} 2=$ moderate), and $\geq 302 \mathrm{~dB} / \mathrm{m}$ (S3 = severe), respectively.

\section{Liver Biopsy}

Considering that screening was being conducted in a predominantly obese population with T2DM at a high risk of having NASH with advanced fibrosis, an ultrasoundguided liver biopsy was considered and discussed with each patient based on clinical risk criteria and, in particular, in those with a VCTE of $\geq 7.0 \mathrm{kPa}$ and AST $\geq 20$ units/L. If the initial VCTE was of $\geq 7.0 \mathrm{kPa}$ and AST $\leq 20$ units/L, a repeat VCTE after $10 \mathrm{~h}$ of fasting was performed (this test was used as the final VCTE for disease prevalence) and laboratories repeated (i.e., AST/ALT and biomarkers of liver fibrosis). Participants were invited to have a liver biopsy if the results were consistent with a moderate-to-severe risk of fibrosis. All patients who underwent a liver biopsy had a VCTE $\geq 8.2 \mathrm{kPa}$. Biopsies were evaluated by a single pathologist who was blinded to the subjects' identity or clinical information (P.B.). Histologic characteristics for the diagnosis of NASH were determined using standard Clinical Research Network criteria (4).

Metabolic Measurements and Fibrosis Diagnostic Panel

We measured fasting plasma glucose, $\mathrm{HbA}_{1 \mathrm{c}}$, lipids, AST, and ALT. For the evaluation of significant liver fibrosis, we used the APRI and FIB-4 as diagnostic scores. We calculated the APRI as follows: $A P R I=(100 \times$ AST/upper limit of normal AST)/platelets $\left(\times 10^{9} / \mathrm{L}\right)(37)$. The APRI was categorized as absence of liver fibrosis if APRI was $<0.5$ or presence of fibrosis if $A P R I \geq 0.5$. We also calculated the FIB-4 as: (age [years] $\times$ AST [units/ L])/(platelet count $\left[10^{9} / \mathrm{L}\right] \times$ square root of ALT [units/L]). Participants with FIB-4 $<1.3$ were considered as being at the lowest risk of advanced liver fibrosis, $\geq 1.67$ to $<2.67$ as having a moderate risk, while those with FIB-4 $\geq 2.67$ were classified as having a high risk of advanced liver fibrosis (F3 or F4). Both APRI and FIB-4 were chosen as diagnostic panels as they have been well-validated and supported by the literature $(4,5)$.

\section{Statistical Analysis}

Data were summarized in percentages for categorical variables and as mean $\pm \mathrm{SE}$ for numeric variables. Categorical variables were compared by performing the $\chi^{2}$ or Fisher exact test. For comparisons between two groups, we performed Kruskal-Wallis or Student $t$ test for numeric variables, depending on variables' distribution. Comparisons among three or more groups were performed with ANOVA (Bonferroni method for post hoc testing) or Kruskal-Wallis test. Pearson or Spearman correlations were used for numerical variables according to their characteristics. A two-tailed $P$ value $<0.05$ was considered to indicate statistical significance. Analyses were performed with JMP Pro 15.0.0 software (SAS Institute Inc., Cary, NC).

\section{RESULTS}

\section{Clinical Characteristics}

A total of 647 participants qualified for the study. A flow diagram is included in
Supplementary Fig. 1. Of those, 72 failed to complete the VCTE evaluation and were excluded, 5 had unreliable FibroScan, and 9 participants were mistakenly included after a secondary review identified that they were on medications in the exclusion criteria. There was no adverse event related to the use of VCTE. The final cohort included data from 561 patients (Table 1). Of the total of 561 patients evaluated by VCTE, 234 (42\%) were done with the M probe and 327 (58\%) with the XL probe. VCTE readings were valid (per the manufacturer's suggestions with at least 10 valid individual measurements) in $99 \%$ of patients, and the few failures were mostly with the XL probe. Within the 561 patients with a valid FibroScan, patients assessed with the $\mathrm{XL}$ probe had a significantly higher BMI than patients measured by the M probe ( $36.3 \pm 4.6$ vs. 29.3 $\left.\pm 5.4 \mathrm{~kg} / \mathrm{m}^{2} ; P<0.0001\right)$. Participants were recruited from general internal medicine ( $21 \% ; n=122)$, family medicine ( $49 \%$; $n=268)$, and endocrinology/diabetes (30\%; $n=171$ ) outpatient clinics.

The BMI distribution included $8 \%$ of lean participants, $26 \%$ of overweight, and $66 \%$ that were obese. The mean fasting plasma glucose was $143 \pm 61 \mathrm{mg} / \mathrm{dL}$, and $\mathrm{HbA}_{1 \mathrm{c}}$ was $7.5 \pm 1.8 \%$, with patients evenly distributed among those with an $\mathrm{HbA}_{1 \mathrm{c}} \geq 7.0 \%$ (52\%) versus $<7.0 \%$ (48\%). Of note, there was no significant difference in the prevalence of steatosis (CAP $\geq 274$ $\mathrm{dB} / \mathrm{m}$; yes/no: $56 \%$ vs. $44 \%$; not significant) by $\mathrm{HbA}_{1 \mathrm{c}}<7.0 \%$ or $\geq 7.0 \%$.

Mean AST and ALT were $21 \pm 10$ units/ $\mathrm{L}$ and $24 \pm 16$ units/L, respectively. Of note, overall, only $6 \%$ and $10 \%$ of patients with steatosis and fibrosis had an AST or ALT $\geq 40$ units/L, respectively (Fig. 1B), suggesting that this cutoff is too high and of limited clinical value to identify these patients in primary care clinics. With a lower cutoff of AST and ALT of $\geq 30$ units/ $L$, it increased to $13 \%$ and $22 \%$, respectively. Finally, $40 \%$ of patients with fibrosis had a plasma AST $\geq 26$ units/L, a cutoff that in prior work from our group helped identify patients at greater risk of clinically significant liver fibrosis (25).

\section{Prevalence of Liver Fibrosis}

The prevalence of suspected liver fibrosis in patients with T2DM was $21 \%$ (i.e., "any fibrosis" or from F1 [LSM $\geq 7.0 \mathrm{kPa}$ ] to F4) (Fig. 1A). When analyzed by severity of fibrosis stages (Fig. 2A), the prevalence of 
Table 1-Patient characteristics

Clinical parameters $(n=561)$

\begin{tabular}{|c|c|}
\hline Age (years) & $60 \pm 11$ \\
\hline Sex (male/female) & $44 / 56$ \\
\hline BMI $\left(\mathrm{kg} / \mathrm{m}^{2}\right)$ & $33.4 \pm 6.2$ \\
\hline Overweight & 26 \\
\hline Obese & 66 \\
\hline \multicolumn{2}{|l|}{ Ethnicity } \\
\hline Caucasian & 57 \\
\hline African American & 30 \\
\hline Hispanics & 7 \\
\hline Asian & 4 \\
\hline Other/not reported & 2 \\
\hline $\mathrm{HbA}_{1 \mathrm{c}}(\%)$ & $7.5 \pm 1.8$ \\
\hline Fasting plasma glucose (mg/dL) & $143 \pm 61$ \\
\hline Systolic blood pressure (mmHg) & $134 \pm 16$ \\
\hline Diastolic blood pressure (mmHg) & $79 \pm 9$ \\
\hline Total cholesterol (mg/dL) & $166 \pm 49$ \\
\hline Triglycerides (mg/dL) & $156 \pm 137$ \\
\hline LDL cholesterol (mg/dL) & $89 \pm 39$ \\
\hline HDL cholesterol (mg/dL) & $47 \pm 12$ \\
\hline AST (units/L) & $21 \pm 10$ \\
\hline ALT (units/L) & $24 \pm 16$ \\
\hline Mean CAP $(\mathrm{dB} / \mathrm{m})$ & $305 \pm 63$ \\
\hline Mean LSM (kPa) & $6.2 \pm 3.4$ \\
\hline \multicolumn{2}{|l|}{ Diabetes medications } \\
\hline Metformin & 76 \\
\hline Sulfonylureas & 24 \\
\hline SGLT2 inhibitors & 7 \\
\hline DPP-4 inhibitors & 14 \\
\hline Insulin & 38 \\
\hline \multicolumn{2}{|l|}{ Lipid-lowering agents } \\
\hline Statins & 67 \\
\hline Fibrates & 4 \\
\hline Ezetimibe & 1 \\
\hline
\end{tabular}

Data are mean \pm SE or \%. DPP-4, dipeptidyl peptidase 4; SGLT2, sodium-glucose cotransporter 2.

mild fibrosis (F1) was $6.5 \%$, moderate fibrosis (F2) $5.6 \%$, severe fibrosis (F3) $6.2 \%$, and cirrhosis $3.0 \%(F 4)$. Only a minority of patients with F2 or higher had an elevated AST or ALT ( $\geq 40$ units/L): AST, $18 \%$ and ALT, $28 \%$. We also analyzed AST and ALT per fibrosis stage:
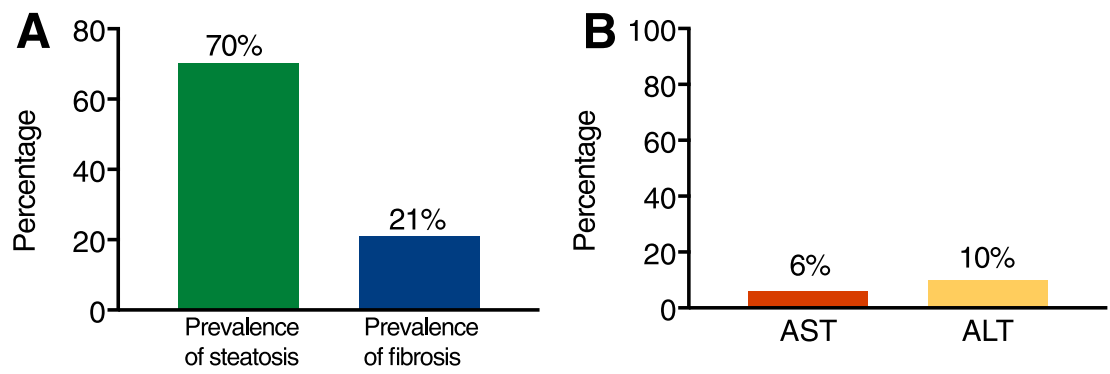

Figure 1-A: Proportion of patients with T2DM screened in the outpatient clinical setting having liver steatosis (measured by CAP) and with liver fibrosis (LSM by VCTE). B: Proportion of patients with steatosis and fibrosis having plasma AST or ALT levels $\geq 40$ units/L. Total number of patients: 561 .
When biochemical diagnostic panels were assessed, APRI was normal in $49 \%$ and in the "indeterminate zone" in $38 \%$ of patients, while positive $(\geq 0.5)$ in only $13 \%$. FIB-4 led to overall similar results, with $57 \%$ of all patients having the panel within the normal range and $38 \%$ in the "indeterminate zone," while $5 \%$ were classified in the advanced fibrosis group (FIB-4 $\geq 2.67$ ).

Patients in whom LSM and APRI and/or FIB-4 suggested liver fibrosis were invited to have a liver biopsy. Among patients at risk for liver fibrosis, 39\% were not interested in additional imaging or confirmatory laboratories (to confirm need for a biopsy), $22 \%$ had an AST $<20$ units/L (criteria for not doing a biopsy as significant fibrosis was highly unlikely), while $6 \%$ declined a liver biopsy after studies suggested a high probability of fibrosis. Another $5 \%$ were on medications (e.g., anticoagulants) or had medical conditions that prevented from performing a biopsy, and $4 \%(n=5)$ did not meet all inclusion/exclusion criteria upon second review and were disqualified from the study (e.g., recently added GLP-1RAs or other medications). Four patients were not biopsied at the principal investigator's discretion, as LSM was between 7.0 and $7.4 \mathrm{kPa}$ and had normal AST/ALT plus a clinical profile not suggestive of being at risk for significant fibrosis. Among those with $L S M \geq 7.0$, 21 had a liver biopsy (all had $L S M \geq 8.2$ $\mathrm{kPa})$. Their profile is summarized in Supplementary Table 1 . The average BMI was $37.1 \pm 6.7 \mathrm{~kg} / \mathrm{m}^{2}, \mathrm{HbA}_{1 \mathrm{c}} 7.5 \pm$ 1.5\%, AST $34 \pm 4$ units/L, and ALT $44 \pm$ 8 units/L. Mean CAP was $351 \pm 38 \mathrm{~dB} / \mathrm{m}$ and LSM $10.3 \pm 2.2 \mathrm{kPa}$. The clinical characteristics among the 63 participants who did not have a liver biopsy were similar to those who were biopsied, but with even more volunteers being obese and with a higher mean LSM (13.4 $\pm 6.4 \mathrm{kPa} ; P=0.06)$ (Supplementary Table 1). Liver fibrosis was confirmed by biopsy in all but two of the patients ( $90 \%$ of those biopsied), who also did not have steatohepatitis.

\section{Prevalence of Liver Steatosis}

The prevalence of NAFLD based on transient elastography was $70 \%$ (CAP $\geq 274$ $\mathrm{dB} / \mathrm{m}$ ) (Fig. 1A). The distribution of mild (S1), moderate (S2), and severe (S3) steatosis was $9 \%, 7 \%$, and $54 \%$, respectively (Fig. $3 A$ ). There was a significant correlation between $\mathrm{BMI}$ and steatosis 

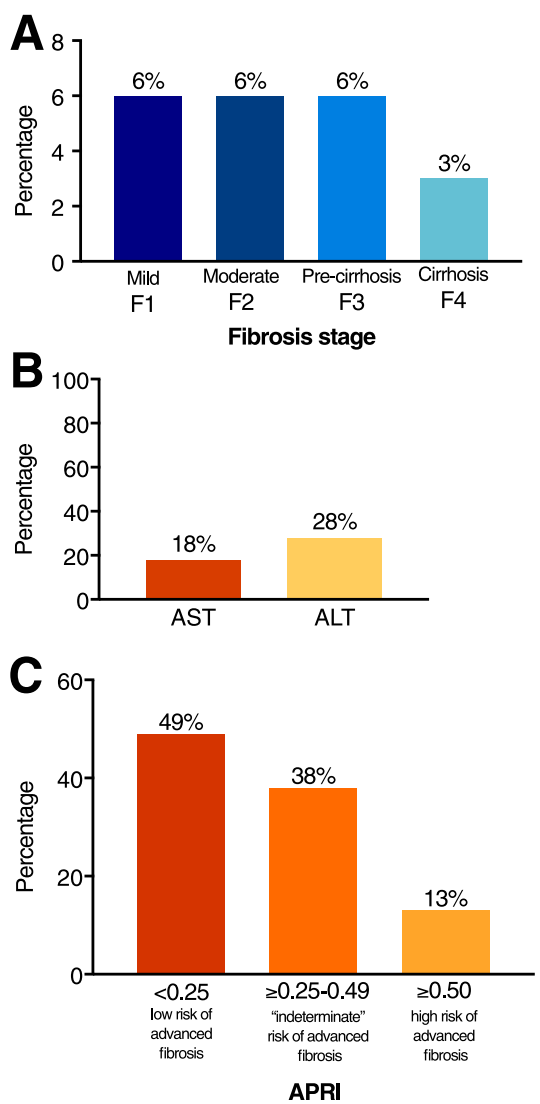

Figure 2-A: Severity of liver fibrosis (LSM) in patients with T2DM screened in the outpatient clinical setting, divided into four stages: mild (F1), moderate (F2), severe or precirrhosis (F3), and cirrhosis (F4). B: Proportion of patients with moderate-to-advanced fibrosis ( $\geq$ F2) having elevated plasma AST or ALT levels. $C$ : Proportion of patients with a low risk, indeterminate risk, or high risk of advanced liver fibrosis by the fibrosis diagnostic panel APRI score. Total number of patients: 561 .

by CAP $\left(r^{2}=0.20 ; P<0.001\right)$ and to a lesser degree between $\mathrm{BMI}$ and liver fibrosis by $\operatorname{LSM}\left(r^{2}=0.12 ; P<0.001\right)$. The proportion of patients with steatosis increased to $40 \%$ with a BMI $\geq 40 \mathrm{~kg} / \mathrm{m}^{2}$. Only $8 \%$ and $13 \%$ of patients with steatosis had AST and ALT levels $\geq 40$ units/L, respectively (Fig. $3 B$ ).

The presence of fibrosis (F1 or higher or $L S M \geq 7.0 \mathrm{kPa}$ ) occurred in $10 \%$ of patients with mild steatosis (S1), $23 \%$ of those with moderate steatosis (S2), and $30 \%$ when steatosis was severe (S3) $(P<$ 0.05 for S1 and S2 vs. S3). Less than $10 \%$ of patients with fibrosis had mild or moderate (S1 or S2) steatosis, compared with $59 \%$ with F1, 81\% with F2, 83\% with F3, and $87 \%$ with F4. Steatosis by CAP with fibrosis by VCTE was present in
62 out of 67 with F2-F3 (93\%) and in 14 out of 15 with F4 (93\%). The greatest discordance with steatosis occurred in those with mild fibrosis: 29 out of 37 (78\%) with S1. However, all had a CAP of $>250 \mathrm{~dB} / \mathrm{m}$, suggesting that they did have some degree of liver fat accumulation.

\section{CONCLUSIONS}

This study is the first of its kind in the U.S. aiming to establish the magnitude of the disease burden in random patients with T2DM seeking regular outpatient care. By determining the extent of the problem and the diagnostic gap despite recent ADA guidelines (6), we hoped to help shape future management strategies. The strengths of the study include: 1) the systematic screening approach of unselected patients in the outpatient setting; 2) having carefully established the presence of NAFLD (i.e., by ruling out by direct patient contact and electronic medical record review all secondary causes of liver disease), something difficult for epidemiological studies based solely on mailed questionnaires or phone interviews; 3) use of a combined pointof-care imaging (transient elastography) and blood biomarker panel approach; and 4) a confirmatory strategy of a liver biopsy in those suspected of advanced liver fibrosis from noninvasive testing. Therefore, the study design offered a robust way to establish the prevalence of NAFLD in patients with T2DM in the "real world." The truly alarming finding was that almost one in six participants (15\%) had unsuspected moderate-to-advanced fibrosis (F2 or higher) (Fig. 2A).

Steatosis was present in almost three out of four patients (Fig. 1A) and was more common in those with higher BMI. A 70\% prevalence in patients with T2DM is higher than in studies based on ultrasound (in which reported prevalence rates are $\sim 55 \%$ ) (3). The higher rate of steatosis by CAP was expected, as the sensitivity of ultrasonography is suboptimal to detect mild-to-moderate steatosis (38), therefore missing patients with mild (S1) or moderate (S2) steatosis but in close agreement with the $54 \%$ of patients who had severe steatosis (S3) (Fig. 3A). Our results also align well to those observed by other investigators using transient elastography in European (26-29) and Asian (30-33) cohorts, as well as with MR-based studies performed in the U.S. (39-41). It is also similar to those recently reported in patients with diabetes from the National Health and Nutrition Examination Survey 20152016 database using plasma steatosis indices, such as the U.S. Fatty Liver Index (23). A higher BMI correlated with worse steatosis and, to a lesser extent, with fibrosis. Most patients with moderateto-advanced fibrosis (F2 or higher) had severe steatosis (S3). A CAP of $\geq 302 \mathrm{~dB} / \mathrm{m}$ (S3) usually corresponds to $\geq 10 \%$ liver fat by MR-based techniques and is consistent with prior work suggesting that more steatosis beyond this level has a modest association with worse steatohepatitis or fibrosis (42). With the limitations of a cross-sectional study, this study suggests a link between the longterm risk of advanced fibrosis and the presence of obesity and "lipotoxicity" $(2,9,43)$.

Increasing evidence suggests that the fibrosis stage at diagnosis is closely associated with future risk of cirrhosis and mortality $(4,5,7,8)$. The finding that $15 \%$ of patients screened and unknown to have NAFLD had moderate-to-advanced fibrosis (F2 or higher) is a key finding that should trigger a call to action by all clinicians taking care of patients with T2DM. In contrast, mild fibrosis (F1) is currently viewed as early stage in the natural history of the disease that most times will not progress to cirrhosis. However, while the determinants of fibrosis progression and regression in NASH are poorly understood (44), in the setting of obesity and T2DM, it is possible that many patients with early disease (F1) are at risk for being "rapid progressors" to more severe liver disease, as suggested in some studies (45-47). That one in six patients with unsuspected disease already had fibrosis supports the notion that patients with obesity and diabetes are at the highest risk and in need of more aggressive screening. These results help explain why $50 \%$ of patients with NASH from cryptogenic cirrhosis have T2DM (48) and diabetes is so prevalent in patients with NASH needing liver transplantation (49). Recent studies highlight the increasing burden of NASH in patients with T2DM (50).

How to best identify patients at risk for liver fibrosis early on in primary care and diabetes clinics remains a challenge but is also an opportunity, as most have a disease stage likely reversible with treatment 

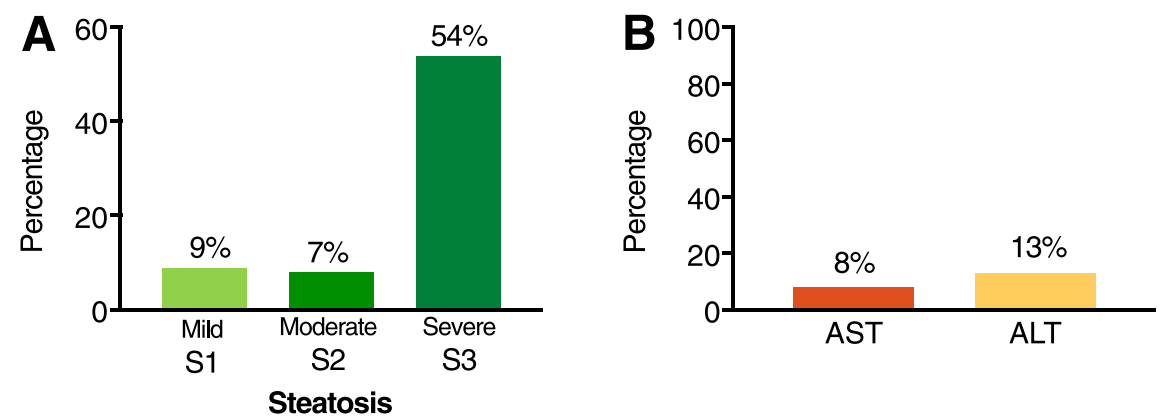

Figure 3-A: Severity of liver steatosis in patients with T2DM screened in the outpatient clinical setting divided into mild (S1), moderate (S2), and severe (S3). B: Proportion of patients with steatosis having elevated plasma AST or ALT levels. Total number of patients: 561.

(F1-F3) (Fig. 2A). Use of APRI or FIB-4 to identify patients with fibrosis was helpful but primarily in the minority of patients with elevated AST or ALT (Figs. $2 B$ and $3 B)$, as expected from their formulas being strongly based on plasma aminotransferases. This is different than in liver clinics, where an elevated AST and ALT are the most common cause for referral. This reality calls for a different diagnostic approach in primary care clinics, where imaging may play a bigger role. The prevalence of moderate-to-advanced fibrosis among patients with diabetes has been usually lower using blood diagnostic panels $(23,26,27,51)$ than in studies based on elastography either from Europe (26-29) or Asia (30-33). Koehler et al. (26) found in a population-based study in Rotterdam that $17.2 \%$ of patients with diabetes and steatosis had moderate-to-advanced fibrosis, more than twofold higher than those with steatosis but without diabetes. Sporea et al. (27) reported an even higher prevalence of moderate-to-advanced fibrosis (21\%) in 524 patients screened by elastography with T2DM from Romania. Prevalence rates of moderate-to-advanced fibrosis have ranged between $17 \%$ and $21 \%$ by elastography-based studies from Hong Kong (32), Vietnam (31), and Malaysia (30). Our results are overall comparable to these earlier studies, although only one prior study in Chinese patients (32) attempted to do a confirmatory liver biopsy if fibrosis was suspected by noninvasive testing. We found that a liver biopsy confirmed the presence of fibrosis in the vast majority of patients and supported the validity of a combined blood and elastography noninvasive approach in the primary care setting. While a limitation is that a minority of patients underwent a liver biopsy, the clinical, laboratory, and imaging characteristics of these patients was similar to those who refused it. Indeed, the nobiopsy group had more obese patients and had a higher mean LSM on elastography, suggestive of perhaps even more severe liver disease and likely to also have significant liver fibrosis (Supplementary Table 1).

There are many agents for NASH undergoing development and some in late stages awaiting FDA approval $(52,53)$. Even today, when there are no Food and Drug Administration-approved agents, early diagnosis is relevant, as reversal of steatohepatitis and even fibrosis are possible by weight loss with lifestyle intervention or bariatric surgery $(9,12)$. In addition, vitamin $E$ has proven effective in patients without diabetes (54). Some medications for the treatment of diabetes allow a dual approach of treating both diabetes and NASH (9-11). Pioglitazone is recommended as an option per current guidelines $(5,6)$ in either patients with $(17,55,56)$ or without $(54,57)$ diabetes. Emerging evidence with GLP-1RAs makes it likely that this class of agents will also play a significant role in the future (58). However, the impact of hyperglycemia in patients with T2DM and NASH remains to be fully established $(2,9,10)$.

In conclusion, this study offers compelling evidence that NASH is a major health problem for patients with T2DM. An early diagnosis is important since there are current interventions that may halt or reverse the disease, with more soon to become available. These results call to more forcefully implement the recent ADA guidelines about identifying hepatic fibrosis in patients with T2DM with steatosis or elevated ALT
(6). Patient and physician awareness of the hepatic and extrahepatic complications of NASH and reversing current diagnosis and treatment inertia will be the only way to avert the looming epidemic of cirrhosis in patients with diabetes.

Acknowledgments. The authors thank the patients, families, and all investigators involved in this study.

Duality of Interest. This study was funded in part by Echosens (Paris, France). K.C. has received research support as principal investigator for the UF from the National Institutes of Health, Cirius Therapeutics, Echosens, Inventiva, Novartis, Novo Nordisk, Poxel, and Zydus Pharmaceuticals and is a consultant for Allergan, Altimmune, Arrowhead Pharmaceuticals, Inc., AstraZeneca, Bristol-Myers Squibb, Boehringer Ingelheim, Coherus BioSciences, Eli Lilly and Company, Fractyl Laboratories Inc., Hanmi Pharmaceutical Co., Ltd., Genentech, Gilead Sciences, Inc., Intercept Pharmaceuticals, Janssen, Pfizer, ProSciento, Inc., Madrigal Pharmaceuticals, and Novo Nordisk. No other potential conflicts of interest relevant to this article were reported.

Author Contributions. R.L. was responsible for patient recruitment and follow-up, data acquisition and interpretation, and writing and editing of the manuscript. F.B. contributed to data analysis and critical revisions of the manuscript. E.G.L., S.S., L.M., J.Mar., D.B., D.P., and N.F. were responsible for patient recruitment and followup and data acquisition and interpretation. S.S., J.B., J.P.R., K.-L.C., G.S., J.Mal., and K.H. were responsible for patient recruitment. P.B. was responsible for pathology reading of liver biopsies. S.K. performed laboratory measurements and data analysis. K.C. contributed to the study design, analysis of the results, and writing and critical revisions of the manuscript. K.C. is the guarantor of this work and, as such, had full access to all of the data in the study and takes responsibility for the integrity of the data and the accuracy of the data analysis.

Prior Presentation. This work was presented at the 80th Scientific Sessions of the American Diabetes Association, 12-16 June 2020. 


\section{References}

1. Budd J, Cusi K. Nonalcoholic fatty liver disease: what does the primary care physician need to know? Am J Med 2020;133:536-543

2. Gastaldelli A, Cusi K. From NASH to diabetes and from diabetes to NASH: mechanisms and treatment options. JHEP Rep 2019;1:312-328

3. Younossi ZM, Golabi P, de Avila L, et al. The global epidemiology of NAFLD and NASH in patients with type 2 diabetes: a systematic review and metaanalysis. J Hepatol 2019;71:793-801

4. Chalasani N, Younossi Z, Lavine JE, et al. The diagnosis and management of nonalcoholic fatty liver disease: practice guidance from the American Association for the Study of Liver Diseases. Hepatology 2018;67:328-357

5. European Association for the Study of the Liver; European Association for the Study of Diabetes; European Association for the Study of Obesity. EASL-EASD-EASO clinical practice guidelines for the management of non-alcoholic fatty liver disease. Diabetologia 2016;59:11211140

6. American Diabetes Association. 4. Comprehensive medical evaluation and assessment of comorbidities: Standards of Medical Care in Diabetes-2020. Diabetes Care 2020;43(Suppl. 1):S37-S47

7. Taylor RS, Taylor RJ, Bayliss S, et al. Association between fibrosis stage and outcomes of patients with nonalcoholic fatty liver disease: a systematic review and meta-analysis. Gastroenterology 2020;158:1611-1625.e12

8. Younossi ZM, Stepanova M, Rafiq N, et al. Nonalcoholic steatofibrosis independently predicts mortality in nonalcoholic fatty liver disease. Hepatol Commun 2017;1:421-428

9. Alkhouri N, Poordad F, Lawitz E. Management of nonalcoholic fatty liver disease: lessons learned from type 2 diabetes. Hepatol Commun 2018;2: 778-785

10. Stefan N, Roden M. Diabetes and fatty liver. Exp Clin Endocrinol Diabetes 2019;127(S 01): S93-S96

11. Stefan N, Häring HU, Cusi K. Non-alcoholic fatty liver disease: causes, diagnosis, cardiometabolic consequences, and treatment strategies. Lancet Diabetes Endocrinol 2019;7:313-324

12. Targher G, Byrne CD, Tilg H. NAFLD and increased risk of cardiovascular disease: clinical associations, pathophysiological mechanisms and pharmacological implications. Gut 2020; 69:1691-1705

13. Bower G, Toma T, Harling L, et al. Bariatric surgery and non-alcoholic fatty liver disease: a systematic review of liver biochemistry and histology. Obes Surg 2015;25:2280-2289

14. Cusi K. Incretin-based therapies for the management of nonalcoholic fatty liver disease in patients with type 2 diabetes. Hepatology 2019;69:2318-2322

15. Klebanoff MJ, Corey KE, Chhatwal J, Kaplan LM, Chung RT, Hur C. Bariatric surgery for nonalcoholic steatohepatitis: a clinical and costeffectiveness analysis. Hepatology 2017;65: 1156-1164

16. Yoo ER, Sallam S, Perumpail BJ, et al. When to initiate weight loss medications in the NAFLD population. Diseases 2018;6:91

17. Cusi K, Orsak B, Bril F, et al. Long-term pioglitazone treatment for patients with nonalcoholic steatohepatitis and prediabetes or type 2 diabetes mellitus: a randomized trial. Ann Intern Med 2016;165:305-315

18. Davison BA, Harrison SA, Cotter G, et al. Suboptimal reliability of liver biopsy evaluation has implications for randomized clinical trials. J Hepatol 2020;73:1322-1332

19. Loomba R, Jain A, Diehl AM, et al. Validation of serum test for advanced liver fibrosis in patients with nonalcoholic steatohepatitis. Clin Gastroenterol Hepatol 2019;17:1867-1876.e3

20. Shima T, Sakai K, Oya H, et al. Diagnostic accuracy of combined biomarker measurements and vibration-controlled transient elastography (VCTE) for predicting fibrosis stage of nonalcoholic fatty liver disease. J Gastroenterol 2020;55:100-112

21. Jacqueminet $S$, Lebray $P$, Morra $R$, et al. Screening for liver fibrosis by using a noninvasive biomarker in patients with diabetes. Clin Gastroenterol Hepatol 2008;6:828-831

22. Kim D, Kim WR, Kim HJ, Therneau TM. Association between noninvasive fibrosis markers and mortality among adults with nonalcoholic fatty liver disease in the United States. Hepatology 2013;57: 1357-1365

23. Repetto E, Barb D, Stokes M, Shankar S, Cusi K. High prevalence of NAFLD in patients with obesity and type 2 diabetes. Abstract A-19-1455EASD. Poster presented at the 55th Annual Meeting of the European Association for the Study of Diabetes (EASD), 16-20 September 2019, Barcelona, Spain

24. Siddiqui MS, Yamada G, Vuppalanchi R, et al. Diagnostic accuracy of noninvasive fibrosis models to detect change in fibrosis stage. Clin Gastroenterol Hepatol 2019;17:1877-1885.e5

25. Bril F, McPhaul MJ, Caulfield MP, et al. Performance of plasma biomarkers and diagnostic panels for nonalcoholic steatohepatitis and advanced fibrosis in patients with type 2 diabetes. Diabetes Care 2020;43:290-297

26. Koehler EM, Plompen EP, Schouten JN, et al. Presence of diabetes mellitus and steatosis is associated with liver stiffness in a general population: the Rotterdam study. Hepatology 2016; 63:138-147

27. Sporea I, Mare R, Popescu A, et al. Screening for liver fibrosis and steatosis in a large cohort of patients with type 2 diabetes using vibration controlled transient elastography and controlled attenuation parameter in a single-center real-life experience. J Clin Med 2020;9:1032

28. Lombardi R, Petta S, Pisano G, et al. FibroScan identifies patients with nonalcoholic fatty liver disease and cardiovascular damage. Clin Gastroenterol Hepatol 2020;18:517-519

29. Mantovani A, Turino T, Lando MG, et al. Screening for non-alcoholic fatty liver disease using liver stiffness measurement and its association with chronic kidney disease and cardiovascular complications in patients with type 2 diabetes. Diabetes Metab 2020;46:296-303

30. Lai LL, Wan Yusoff WNI, Vethakkan SR, Nik Mustapha NR, Mahadeva S, Chan WK. Screening for non-alcoholic fatty liver disease in patients with type 2 diabetes mellitus using transient elastography. J Gastroenterol Hepatol 2019;34: 1396-1403

31. Tuong TTK, Tran DK, Phu PQT, Hong TND, Dinh TC, Chu DT. Non-alcoholic fatty liver disease in patients with type 2 diabetes: evaluation of hepatic fibrosis and steatosis using FibroScan. Diagnostics (Basel) 2020;10:159

32. Kwok R, Choi KC, Wong GL, et al. Screening diabetic patients for non-alcoholic fatty liver disease with controlled attenuation parameter and liver stiffness measurements: a prospective cohort study. Gut 2016;65:1359-1368

33. Chen K, Sng WK, Quah JH, et al. Clinical spectrum of non-alcoholic fatty liver disease in patients with diabetes mellitus. PLoS One 2020; 15:e0236977

34. de Lédinghen V, Vergniol J. Transient elastography (FibroScan). Gastroenterol Clin Biol 2008;32(Suppl. 1):58-67

35. Vuppalanchi R, Siddiqui MS, Van Natta ML, et al.; NASH Clinical Research Network. Performance characteristics of vibration-controlled transient elastography for evaluation of nonalcoholic fatty liver disease. Hepatology 2018;67: 134-144

36. Eddowes PJ, Sasso $M$, Allison $M$, et al. Accuracy of FibroScan controlled attenuation parameter and liver stiffness measurement in assessing steatosis and fibrosis in patients with nonalcoholic fatty liver disease. Gastroenterology 2019;156:1717-1730

37. Castera L, Friedrich-Rust M, Loomba R: Noninvasive assessment of liver disease in patients with nonalcoholic fatty liver disease. Gastroenterology 2019;156:1264-1281.e4

38. Bril F, Ortiz-Lopez C, Lomonaco R, et al. Clinical value of liver ultrasound for the diagnosis of nonalcoholic fatty liver disease in overweight and obese patients. Liver Int 2015; 35:2139-2146

39. Doycheva I, Cui J, Nguyen P, et al. Noninvasive screening of diabetics in primary care for NAFLD and advanced fibrosis by MRI and MRE. Aliment Pharmacol Ther 2016;43:8395

40. Portillo-Sanchez $P$, Bril F, Maximos $M$, et al. High prevalence of nonalcoholic fatty liver disease in patients with type 2 diabetes mellitus and normal plasma aminotransferase levels. J Clin Endocrinol Metab 2015;100:22312238

41. Cusi K, Sanyal AJ, Zhang S, et al. Non-alcoholic fatty liver disease (NAFLD) prevalence and its metabolic associations in patients with type 1 diabetes and type 2 diabetes. Diabetes Obes Metab 2017;19:1630-1634

42. Bril F, Barb D, Portillo-Sanchez P, et al. Metabolic and histological implications of intrahepatic triglyceride content in nonalcoholic fatty liver disease. Hepatology 2017;65: 1132-1144

43. Cusi K. Role of obesity and lipotoxicity in the development of nonalcoholic steatohepatitis: pathophysiology and clinical implications. Gastroenterology 2012;142:711-725e6

44. Schuppan D, Surabattula R, Wang XY. Determinants of fibrosis progression and regression in NASH. J Hepatol 2018;68:238-250

45. Pais R, Charlotte F, Fedchuk L, et al.; LIDO Study Group. A systematic review of follow-up biopsies reveals disease progression in patients with non-alcoholic fatty liver. J Hepatol 2013;59: 550-556

46. Ekstedt M, Franzén LE, Mathiesen UL, et al. Long-term follow-up of patients with NAFLD and elevated liver enzymes. Hepatology 2006;44: 865-873 
47. Bril F, Kalavalapalli S, Clark VC, et al. Response to pioglitazone in patients with nonalcoholic steatohepatitis with vs without type 2 diabetes. Clin Gastroenterol Hepatol 2018;16:558-566.e2

48. Lee WG, Wells $\mathrm{Cl}$, McCall JL, Murphy R, Plank LD. Prevalence of diabetes in liver cirrhosis: a systematic review and meta-analysis. Diabetes Metab Res Rev 2019;35:e3157

49. Golabi $P$, Bush $H$, Stepanova $M$, et al. Liver transplantation (LT) for cryptogenic cirrhosis (CC) and nonalcoholic steatohepatitis (NASH) cirrhosis: data from the scientific registry of transplant recipients (SRTR): 1994 to 2016. Medicine (Baltimore) 2018;97:e11518

50. Younossi ZM, Tampi RP, Racila A, et al. Economic and clinical burden of nonalcoholic steatohepatitis in patients with type 2 diabetes in the U.S. Diabetes Care 2020;43:283-289
51. Morling JR, Fallowfield JA, Guha IN, et al.; Edinburgh Type 2 Diabetes Study investigators. Using non-invasive biomarkers to identify hepatic fibrosis in people with type 2 diabetes mellitus: the Edinburgh Type 2 Diabetes Study. J Hepatol 2014;60:384-391

52. Friedman SL, Neuschwander-Tetri BA, Rinella M, Sanyal AJ. Mechanisms of NAFLD development and therapeutic strategies. Nat Med 2018;24:908-922

53. Alkhouri N. NASH and NAFLD: emerging drugs, therapeutic targets and translational and clinical challenges. Expert Opin Investig Drugs 2020;29:87

54. Sanyal AJ, Chalasani N, Kowdley KV, et al.; NASH CRN. Pioglitazone, vitamin E, or placebo for nonalcoholic steatohepatitis. N Engl J Med 2010; 362:1675-1685
55. Bril F, Biernacki DM, Kalavalapalli S, et al. Role of vitamin E for nonalcoholic steatohepatitis in patients with type 2 diabetes: a randomized controlled trial. Diabetes Care 2019;42:14811488

56. Belfort R, Harrison SA, Brown K, et al. A placebo-controlled trial of pioglitazone in subjects with nonalcoholic steatohepatitis. N Engl J Med 2006;355:2297-2307

57. Aithal GP, Thomas JA, Kaye PV, et al. Randomized, placebo-controlled trial of pioglitazone in nondiabetic subjects with nonalcoholic steatohepatitis. Gastroenterology 2008;135:11761184

58. Dhir G, Cusi K. Glucagon like peptide-1 receptor agonists for the management of obesity and non-alcoholic fatty liver disease: a novel therapeutic option. J Investig Med 2018;66:7-10 